\title{
Prevention of Type 2 Diabetes - Lessons we have Learnt for Implementation
}

Author

Affiliation
P. E. H. Schwarz ${ }^{1}$, M. Peltonen ${ }^{2}$

${ }^{1}$ III ${ }^{\circ}$ Medical Clinic, Carl Gustav Carus Medical School, Dresden University of Technology, Dresden, Germany ${ }^{2}$ National Public Health Institute, Department of Health Promotion and Chronic Disease Prevention, Diabetes Unit, Helsinki, Finland

\section{Key words}

- Prevention of type 2 diabetes

- quality management

- intervention concept

- implementation

- public health

- FINDRSIC received 29.03.2007

accepted 09.05.2007

\section{Bibliography}

DOI 10.1055/s-2007-985175

Horm Metab Res 2007;

39: 636-641

(c) Georg Thieme Verlag KG

Stuttgart · New York

ISSN 0018-5043

\section{Correspondence}

\section{Dr. med. P. E. H. Schwarz}

III ${ }^{\circ}$ Medical Clinic

Carl Gustav Carus Medical

School

Dresden University of

Technology

Fetscherstrasse 74

01307 Dresden

Germany

Tel.: + 49/351/4582715

Fax: +49/351/4587319

peter.schwarz@uniklinikum-

dresden.de

\section{Abstract}

$\nabla$

The challenge today is to implement successful diabetes prevention programs in general health care. Even if not all questions for the prevention of diabetes are answered, we have today overwhelming evidence that diabetes can be prevented or delayed in high risk population through lifestyle modification or pharmacological interventions. This information has to be translated now into well-defined strategies for screening and treating high risk population in daily practice. It is necessary then to develop and implement prevention programs into clinical practice considering scientific aspects and

\section{The Problem Diabetes}

$\nabla$

The dramatic increase in newly diagnosed cases of type 2 diabetes has developed into a major public health concern in this century [1]. Having diabetes means having a significantly reduced quality of life and reduced life expectancy [2]. Furthermore, diabetes and impairment of glucose tolerance is increasingly appearing amongst the elderly and recently also in younger people with a most sudden increase in the age group of below 30 years [3]. This results in an increasing number of people being affected by diabetes mellitus in their working age making diabetes to an economic factor. The continuously decreasing age of diagnosis makes a longer and intensified medical treatment necessary due to the increase in medical standards [4-5].

Recent large meta-analysis shows that more than half of all Europeans will suffer from hyperglycemia and diabetes during their lifetime [6-10]. This increase in incidence has resulted in dramatically escalating rates of complications of the disease, especially cardiovascular complications [6]. Recent data from the Framingham study practical requirements during implementation. While translating the scientific evidence into population based intervention strategies more and more questions arise, mostly related to economic and structural requirements during implementation. At the end, giving the right answers to these questions will decide about the success of implemented prevention strategies. Here, we will try to answer some of the questions which arose during the implementation of a prevention management concept into clinical practice. We focus on the development of a structured prevention management program which will enable implementation of diabetes prevention into clinical practice.

show that the proportion of cardiovascular disease due to diabetes has increased over the past 50 years, and the increasing prevalence of diabetes could reverse the decreasing trend in coronary heart disease mortality [11]. Hence, the increased costs due to diabetes are a significant burden for society. All of the described factors increase the economical, medical and social burden of diabetes mellitus exponentially. After diagnosis of diabetes mellitus there is - by now - no cure of the disease - only treatment. The real cure of diabetes is the effective primary prevention.

\section{Risk factors are known $\nabla$}

Type 2 diabetes is a complex metabolic disease developing in genetically susceptible individuals as a result of environmental and lifestyle risk factors [12-19]. These risk factors are well-known: obesity, central adiposity, physical inactivity, and unhealthy diet. The more prevalent sedentary lifestyle and the globalization of "fast" and overly rich nutrition, the more people will develop risk 
factors for diabetes mellitus [20]. Therefore, diabetes is giving rise to excessive rates of heart disease, stroke, peripheral vascular disease, renal and eye diseases, neurological and mental problems [21]. While the age at onset of diabetes is lower, its complications will appear earlier [22]. Due to this excessive rate of complications diabetes is a major cause of morbidity and contributes significantly to premature mortality in all European countries.

\section{Lifestyle modification to prevent diabetes \\ $\nabla$}

The most efficient way to manage diabetes and its complications is to prevent diabetes from developing. Fortunately, recent studies have convincingly demonstrated that prevention of type 2 diabetes is possible [23-26]. The best method of intervention for preventing diabetes is still not clear, but there is overwhelming evidence that diabetes can be prevented or delayed in high risk population through lifestyle modification or pharmacological interventions.

A study from Da Qing, China, looked at the effects of diet and exercise in preventing diabetes in Chinese patients with impaired glucose tolerance (IGT). Risk reductions in diabetes were $31 \%$ with diet, $46 \%$ with exercise, and $42 \%$ with diet plus exercise [26].

In the Diabetes Prevention Study (DPS) in Finland, significant reduction in progression to diabetes was observed as $522 \mathrm{mid}$ dle-aged obese subjects with IGT were randomized to receive either diet and exercise counseling (control group) or intensive individualized instruction on weight reduction, food intake, and guidance on increasing physical activity (intervention group) [24]. After an average follow-up of 3.2 years, there was a $58 \%$ relative reduction in the incidence of diabetes in the intervention group compared with the control subjects. Interestingly strong correlation was also seen between the progression to diabetes and the ability of the participants to achieve one or more of the following core goals to lose weight (goal of $5.0 \%$ weight reduction), reduce fat intake (goal of $<30 \%$ of calories), reduce saturated fat intake (goal of $<10 \%$ of calories), increase fiber intake (goal of $\geq 15 \mathrm{~g} / 1000 \mathrm{kcal}$ ), and exercise (goal of $>150 \mathrm{~min} /$ week) [27]. Recently it was shown that the lifestyle intervention resulted in sustained lifestyle changes and a reduction in diabetes incidence, which were maintained for years after the individual lifestyle counseling was stopped [28].

The largest study up to now has been the Diabetes Prevention Program (DPP) [23]. This study examined 3234 patients with IGT and followed them for an average of 2.8 years. The risk reduction from lifestyle modification was identical to that seen in the Finnish study: $58 \%$. The lifestyle intervention consisted of a minimum of 150 minutes of physical activity per week, and resulted in a $7 \%$ reduction in weight. The DPP also included a group that was assigned to metformin $850 \mathrm{mg}$ twice daily reaching a significant risk reduction of $31 \%$. On average, $50 \%$ of the lifestyle group achieved the goal of $\geq 7 \%$ weight reduction, and $74 \%$ maintained at least $150 \mathrm{~min} /$ week of moderately intense activity. No serious side effects were seen in any group [29].

In the Indian Diabetes Prevention Program, the effect of lifestyle intervention alone, metformin, or a combination of both was tested to be effective to prevent diabetes in persons with IGT [30]. Interestingly the effect of all three strategies was very similar. The relative risk reduction was $28.5 \%$ with lifestyle inter- vention, $26.4 \%$ with metformin and $28.2 \%$ with the combination of both compared with the control group.

\section{Drug treatment to prevent diabetes \\ $\nabla$}

Other studies tested primary or secondary drug therapy to prevent or delay diabetes. In the STOP-NIDDM trial participants with IGT were randomized to receive either acarbose or a placebo. After a mean follow-up of 3.3 years, a $25 \%$ relative risk reduction in progression to diabetes was observed in the acarbose-treated group compared with the placebo group [25]. In the Troglitazone in Prevention of Diabetes (TRIPOD) study the thiazolidinedione troglitazone treatment was associated with a $56 \%$ relative reduction in progression to diabetes. Furthermore it was shown in the XENDOS trial that orlistat [31] significantly decreased progression of impaired glucose tolerance to diabetes by $52 \%$. In the recently finished DREAM Study with over 5000 patients rosiglitazone reduced the risk of developing type 2 diabetes by $62 \%$ relative to placebo among people at high risk of developing type 2 diabetes [32], but the ACE inhibitor ramipril, however, did not reduce the likelihood of progression to diabetes [33]. Other secondary studies showed that ACE inhibitors and angiotensin receptor blockers were associated with reductions in the incidence of newly diagnosed diabetes by $27 \%$ and $23 \%$ [34] as well as pravastatin by $30 \%$ and estrogen/progesterone by $35 \%$ [35]. For some people, medication is part of an overall plan for diabetes prevention but it appears to be that drug therapy to prevent or delay diabetes is less beneficial than lifestyle changes.

This information now has to be translated into well-defined strategies for screening and treating high risk population in clinical practice. While these findings offer the evidence-base for the development of community-based prevention strategies [36], it is necessary to develop and implement prevention programs into clinical practice considering scientific aspects and practical requirements during implementation [37].

While translating the scientific evidence into population based intervention strategies more and more questions arise, mostly related to economic and structural requirements during implementation. At the end giving the right answers to these questions will decide about the success of implemented prevention strategies.

\section{What are the management aims in diabetes prevention? \\ $\nabla$}

The prevalence of diabetes is increasing in epidemic proportion worldwide and it is becoming a major burden for the health care system. With a better understanding of the pathogenesis of type 2 diabetes, the concept of primary prevention has emerged and we have overwhelming evidence that we can prevent or delay the progression to diabetes. Prevention of the disease is our only chance to alleviate the ever growing burden of diabetes mellitus. Knowing this, our first aim is to reduce the incidence of diabetes among those who are at high risk. Further, the effect should be maintained over a long time, and this should ultimately reduce the associated burden of the disease in terms of micro- and macrovascular complications.

To achieve these goals it is necessary to answer the following questions: 1 . Who is at risk for diabetes; 2 . How do people at risk 
receive the necessary information and motivation to change lifestyle - and what intervention is the best one; and 3. What is the best way to maintain lifestyle changes over a long time [38]? Additionally the potential intervention should be accessible with acceptable effort in the general population on a national level reaching people at risk for diabetes.

Based on this we can define 3 management aims in diabetes prevention in the following way:

- Persons with increased diabetes risk are found before disease onset;

- Persons at risk for diabetes are getting an intervention to prevent diabetes; and

- These persons will not develop diabetes or in case of developing the disease, diabetes is detected early and treated with adequate therapy (secondary prevention).

Additionally the management concept should be accessible with acceptable effort in the general population on a national level reaching people at risk for diabetes. Furthermore it is necessary to take into consideration the reimbursement system in Germany and issues of professionalism - to decide who is the professional to carry out the lifestyle intervention [38,39].

It is unrealistic to believe that the increasing trend in the prevalence of diabetes can be stopped focusing solely on high risk subjects. A realistic model of action is a program that achieves over time a proportionally slower increase of diabetes incidence with the intervention, and is able to maintain metabolic changes after discontinuation of the intervention [39]. In addition, a population strategy aiming at promoting health of the entire population by means of healthy diet and physical activity is required to reduce the number of high risk subjects most likely to develop diabetes.

\section{Which intervention concept is necessary? \\ $\nabla$}

For the implementation of a prevention program it is necessary to find a way to translate lessons learned from the prevention studies into a management concept which reaches similar efficiency in lifestyle changes as in the mentioned studies and still being practical to manage also a large number of participants. In the mentioned studies the intervention followed a curricular intervention and was finished after a certain time. The final success in reaching a lifestyle change was related to the degree the participants were able to successfully include lifestyle changes in the daily routine. Knowing this, future prevention management concepts should follow a 3-step intervention plan:

- Identification of the individuals at high risk to develop type 2 diabetes;

- Intensive intervention based on individual choice; and

- Continuous intervention for motivation maintenance and evaluation.

It is an important option to focus the prevention management on group interventions. The intention is to motivate the person at risk to self-manage the diabetes risk and the lifestyle changes with assistance. Whether group interventions are more costeffective than individualized interventions remains to be shown.

\section{Whom to intervene?}

$\nabla$

It is important to address as exactly as possible regarding who should receive an intervention. It is not only a financial issue but also part of our medical responsibility to treat persons who trust the medical professional. The major question is how many false positive screened individuals one accepts to receive an intervention. This discussion should be focused on the kind and the intensity of the intervention. More false positives (for diabetes risk) are accepted with more general a lifestyle advice. If someone accumulates risk factors like adiposity or high blood pressure he will benefit from the intervention, even if he was not considered as with diabetes risk. On the other hand interventions which have sometimes potential serious side effects like drug intervention, and false positive cases in the screening are not acceptable. For diabetes prevention programs the discussion focuses on persons with IGT/IFG or also persons with increased diabetes risk before they have IGT/IFG. Potentially, the preventive effect of interventions aimed at persons with elevated risk while they still are normoglycemic might be higher than for people who already have IGT/IFG. This has implications on who is eligible for intervention.

\section{How to find the persons at risk? $\nabla$}

Strategies to identify persons at high risk for type 2 diabetes were issues of controversy in the past [40]. The effort performed to identify subjects which fulfill inclusion criteria for the larger prevention studies consumes large scale resources and is difficult to realize for a large number of persons. Aiming at including not only persons with elevated diabetes risk but also persons with IGT/IFG made it necessary to use a test that identifies persons at disease risk. An efficient test should also recognize persons having a predictive diabetes risk with a good ratio between specificity and sensitivity, should be simple to handle, transparent to both sides (physician and the affected person), widely accessible, and basically cost free. Furthermore the test should intend an empowerment as feeling "being at risk" and not "being sick".

The Finnish Diabetes Risk Score (FINDRISC) fulfils the above criteria [41] and can be an ideal tool to find person with increased diabetes risk. The FINDRISC can be used as a self-administered test to screen subjects at high risk for type 2 diabetes. It can also be used in the general population and clinical practice to identify undetected T2D, AGT and the metabolic syndrome [42]. This questionnaire comprises validated eight items [41] and it is easy to make the score available widely via the internet, distribute information material by the health care and social institution, and maintaining occupational health care and public health. At the end of the questionnaire a contact opportunity is given using a local telephone or internet address for contacting a prevention manager. Even if the resonance of a single screening shot is low $(0.5 \%)$ it is very easy to increase the redundancy of placing the FINDRISC for screening.

Of the tools currently available, FINDRISC is perhaps the most accurate and widely used. It is the ideal tool to be used in primary prevention programs, because it is simple to understand for lay people, does not require laboratory data and can be applied on population level. FINDRISC is playing a central role in the Finnish Diabetes Prevention Program, which was initiated in 2003 and is due to run until 2010 [42]. Individuals with a FINDRISC score between 7 and 14 receive advice on lifestyle changes, while those with scores greater than 15 are given an OGTT to identify any who have undiagnosed type 2 diabetes requiring more intensive intervention. The FINDRISC is also used as the 
screening instrument in the German National Diabetes Prevention Program [43]. Individuals with a score below 11 will receive written information about healthy diet and exercise benefits. Subjects with a score between 11 and 20 will be encouraged to participate in an intervention program performed in group sessions. Persons having a score of 21 or greater get the recommendation to visit a medical doctor for diabetes diagnosis or exclusion. If diabetes is excluded these persons can participate in the intervention program. The differences in the scoring for diabetes are based on the reimbursement system and the structural implementation. The intervention is much more light in Finland so that the people are earlier (score 15) transferred to the medical system as in Germany where the people can receive a structured intervention, which makes a later referral possible. In addition, FINDRISC will also be used in "Diabetes in Europe: Prevention using Lifestyle, physical Activity and Nutrition intervention (DE-PLAN)", an EU-funded public health project to develop a European diabetes prevention management strategy. FINDRISC will form the basis of a low-cost screening program to identify individuals with high diabetes risk. The presence of prediabetes or undiagnosed type 2 diabetes can then be confirmed by administering an OGTT [40].

\section{How to deliver intervention? \\ $\nabla$}

Another key question to implement a nationwide prevention program of type 2 diabetes is: Who should deliver the intervention? Addressing risk factors for a disease like insulin resistance and obesity is a medical responsibility, but addressing physical inactivity and dietary changes in persons who are still "healthy" is not primarily physicians' responsibility [39]. Furthermore even motivated physicians typically have limited experience in training lifestyle intervention, and often they have inadequate access to the resources needed to support lifestyle intervention. Therefore primary prevention of type 2 diabetes raises several issues related to integration of lifestyle intervention into clinical or preclinical practice. Fortunately in most countries providers for exercise, nutritional and motivational counseling exist with high professionalism [44]. They can be used after a short additional training to deliver the intervention if adequate quality control and evaluation is established. Another concept is the implementation of "prevention managers" who work as specialized providers and coordinators of the intervention [38]. Currently the EU public health research project "IMAGE "Development and Implementation of a European Guideline and Training Standards for Diabetes Prevention" is developing structures for a European prevention management concept including a curriculum for the training of prevention managers funded by the Commission of the European Communities, Directorate C Public Health. The prevention managers can be recruited from existing health care providers like psychologists, dieticians, diabetes educators, physical trainers, physiotherapists, and persons attending an additional training to become a prevention manager if they accept a continuous quality control and evaluation of their work. The advantage is that an existing infrastructure can be used to spread the intervention. Following this concept the physician works as a supervising partner of the prevention manager if a professional diagnosis (diabetes, CHD) is needed [38]. The prevention manager will be a new category of health interventionist to deliver and manage long term lifestyle intervention in the large number of persons who would be eligible for these services [44]. As coordinating centre a central institution for prevention management should manage the program and organize the quality control [38].

\section{What intensity of the intervention is necessary?} $\checkmark$

The prevention studies known today $[23-26,30]$ were performed with different study designs and intervention procedures, different observation time and also the initial hypothesis followed different hypothetical action models [45] and intervention protocols with various intensities [38]. In the DPP an enormous effort was taken to prevent diabetes [23], but the result was very similar to the less intensive intervention performed in the Diabetes Prevention Study from Finland [24]. Furthermore in the Finnish study the intervention resulted in sustained lifestyle changes and a reduction in diabetes incidence, even after the individual lifestyle counseling was stopped. Finally the intervention success in diabetes prevention was related to the success in achieving the intervention goals of weight loss, reduced intake of total and saturated fat and increased intake of dietary fiber, and increased physical activity in the DPS and weight loss and increased physical activity in the DPP [46]. This could lead to the conclusion that the intervention time is secondary - intervention quality focusing on sustained achievement of intervention goals would be the primary goal [28]. Then there is a good chance that less intensive lifestyle programs also are effective in changing lifestyle [44]. One example for less intensive intervention is the example of Finland where the FINDRISC itself is seen already as a minimal intervention. The score has information about diabetes risk factors and possibilities to prevent the disease, and thus it serves as a method to increase the awareness of the disease.

\section{How to control quality of the intervention? \\ $\checkmark$}

The key factors in performing high quality intervention are continuous evaluation and quality control [47]. Therefore a continuous system of quality management and reporting is necessary to install. This can become a key criterion for the success or failure of the intervention. If a program is implemented on nationwide basis the individual success of the participating persons and a long term success of the intervention needs to be reported. To establish such a health reporting in the medical system can easily consume all resources provided. Therefore the long term measurement of blood pressure and waist circumference could be chosen for quality control and an independent evaluation and quality control. Prevention of diabetes and the metabolic syndrome means reduction of metabolic risk factors like blood pressure and waist circumference. In the mentioned prevention studies they were associated with the intervention effect and they are easy to standardize and measure without costly laboratory diagnostics. Collected on a regular basis, both parameters can give a good evaluation about the risk and lifestyle changes $[48,49]$. With such a procedure a transparent feedback is possible both to the person receiving the intervention and the prevention manager. The advantages of the quality control based on the intervention effect will make a successful individual evaluation for the participating persons possible, so that nonresponders can be identified early, who are then eligible for a booster intervention [38]. 


\section{How to maintain the effect?}

Several investigations in the past have shown that, time-limited intervention to change lifestyle fail to reach a continuous lifestyle change $[38,39]$. It is typically human to be activated and motivated for a short period of time, even with a high personal involvement, but failing to maintain further [45]. Still, in the extended follow-up of the Finnish Diabetes Prevention Study, beneficial lifestyle changes and the corresponding reduction in diabetes risk were sustained over a long period of time [42]. Establishing a long term motivation to maintain lifestyle changes to prevent diabetes needs to be the core part of the prevention management concept. Therefore, after the initial intervention it is necessary to establish a bundle of interventions providing a regular contact with a minimum of 4 weeks intervals to strengthen motivation of the participating persons. This can include written "newsletters", telephone counseling and boostering, assisted problem management, continuous support including websites, regular interactive email newsletters, optional booster sessions, and assistance to explore the environment for physical activity. Furthermore, a regular quality control including blood pressure and waist circumference measurement is ideal for personal feedback assisting a sustained lifestyle change $[38,39]$.

\section{Conclusion}

$\nabla$

The only way to reduce the personal and socio-economic burden of diabetes and its associated complications is the prevention of diabetes. The compeling scientific evidence supports primary prevention of diabetes by lifestyle intervention and translations of the study strategies into national prevention programs. Still, it is currently not clear how to implement these intervention methods with maintained effectiveness into primary health care system where resources are scarce. The implementation of diabetes prevention programs will require an integrated, international approach if we are to see significant reduction in the premature morbidity and mortality it causes. We must accept that diabetes risk is not a disease; it is a symptom of a much larger problem - the adaptation of our metabolism to sedentary globalization [51]. Diabetes is likely to remain a huge threat to public health in the years to come. Therefore affordable strategies and quality controlled programs are needed for primary prevention. The health intervention may not be limited to diabetes alone, it should have a potential to prevent a great number of health conditions, not only for those related to the metabolic syndrome but also for diseases like cardiovascular disease, certain cancer types, and osteoporosis. Integration of lifestyle intervention into current health care systems will require a simple network of prevention managers and physicians to provide effective programs of lifestyle intervention and to implement quality controlled prevention strategies. An urgent priority is therefore to establish guidelines for the prevention of type 2 diabetes, including lifestyle and pharmaco-prevention.

\section{References}

1 Zimmet P, Alberti KG, Shaw J. Global and societal implications of the diabetes epidemic. Nature 2001; 414: 782-787

2 Haffner SM. Can reducing peaks prevent type 2 diabetes: implication from recent diabetes prevention trials. Int J Clin Pract Suppl 2002; 129: $33-39$
3 Nestle M. Preventing childhood diabetes: the need for public health intervention. Am J Public Health 2005; 95: 1497-1499

4 Schwarz PE. Congress report from the American Diabetes Association $66^{\text {th }}$ Annual Scientific Sessions in Washington, USA. Exp Clin Endocrinol Diabetes 2006; 114 (10): 605-610

5 Schwarz PE. Report from the Congress of the American Diabetes Association (ADA): Orlando 2005 -65th Annual Scientific Sessions in San Diego, CA, USA, June 10th-14th 2005. Exp Clin Endocrinol Diabetes 2005; 113 (8): 475-479

6 Valensi P et al. Pre-diabetes essential action: a European perspective. Diabetes Metab 2005; 31: 606-620

7 Schwarz PE, Bornstein SR. Pre-diabetes and metabolic syndrome in Germans. Horm Metab Res 2006; 38: 359

8 Kanauchi M et al. New "pre-diabetes" category and the metabolic syndrome in Japanese. Horm Metab Res 2005; 37: 622-626

9 Diamantopolous EJ et al. Metabolic syndrome and prediabetes identify overlaping but not identical populations. Exp Clin Endocrinol Diabetes 2006; 114 (7): 377-383

10 Haslbeck KM et al. The AGE/RAGE/NF-(kappa)B pathway may contribute to the pathogenesis of polyneuropathy in impaired glucose tolerance (IGT). Exp Clin Endocrinol Diabetes 2005; 113 (5): 288-291

11 Fox CS et al. Increasing cardiovascular disease burden due to diabetes mellitus: the Framingham Heart Study. Circulation 2007; 115: 1544-1550

12 Schwarz PE et al. Haplotypes in the promoter region of the ADIPOQ gene are associated with increased diabetes risk in a German caucasian population. Horm Metab Res 2006; 38: 447-451

13 Gouni-Berthold I et al. The K121Q polymorphism of the plasma cell glycoprotein-1 gene is not associated with diabetes mellitus type 2 in German Caucasians. Horm Metab Res 2006; 38: 524-529

14 Fisher $E$ et al. Preliminary evidence of FABP2 A54T polymorphism associated with reduced risk of type 2 diabetes and obesity in women from a German cohort. Horm Metab Res 2006; 38: 341-345

15 Kiessling A, Ehrhart-Bornstein M. Transcription factor 7-like 2 (TCFL2) - a novel factor involved in pathogenesis of type 2 diabetes. Comment on: Grant et al. Nature Genetics 2006, Published online 15 January 2006. Horm Metab Res 2006; 38: 137-138

16 Hoffmann $K$ et al. A German genome-wide linkage scan for type 2 diabetes supports the existence of a metabolic syndrome locus on chromosome $1 \mathrm{p} 36.13$ and a type 2 diabetes locus on chromosome 16p12.2. Diabetologia 2007; 50: 1418-1422

17 Tsuchiya $T$ et al. Association of the calpain-10 gene with type 2 diabetes in Europeans: results of pooled and meta-analyses. Mol Genet Metab 2006; 89: 174-184

18 Trajkovski $M$ et al. Genes of type 2 diabetes in Beta cells. Endocrinol Metab Clin North Am 2006; 35: 357-369

19 Schwarz PE et al. Hypoadiponectinemia is associated with progression toward type 2 diabetes and genetic variation in the ADIPOQ gene promoter. Diabetes Care 2006; 29: 1645-1650

20 Thamer $C$ et al. Beta cell function, insulin resistance and plasma adiponectin concentrations are predictors for the change of postprandial glucose in non-diabetic subjects at risk for type 2 diabetes. Horm Metab Res 2006; 38: 178-182

21 Hiltunen LA. Does glucose tolerance affect elderly persons' balance, gait or muscle strength? Cent Eur J Public Health 2001; 9: 22-25

22 Schutte AE, O'Dea K, Schwarz PE. Could statistical adjustments for age mask the insulin-blood pressure relationship? Diabetes Res Clin Pract 2006; 72: 104-107

23 Knowler WC et al. Reduction in the incidence of type 2 diabetes with lifestyle intervention or metformin. N Engl J Med 2002; 346: 393-403

24 Tuomilehto $\mathrm{J}$ et al. Prevention of type 2 diabetes mellitus by changes in lifestyle among subjects with impaired glucose tolerance. $\mathrm{N}$ Engl J Med 2001; 344: 1343-1350

25 Chiasson JL et al. Acarbose for prevention of type 2 diabetes mellitus: the STOP-NIDDM randomised trial. Lancet 2002; 359: 2072-2077

26 Pan XR et al. Effects of diet and exercise in preventing NIDDM in people with impaired glucose tolerance. The Da Qing IGT and Diabetes Study. Diabetes Care 1997; 20: 537-544

27 American Diabetes Association and National Institute of Diabetes, Digestive and Kidney Diseases. The prevention or delay of type 2 diabetes. Diabetes Care 2002; 25: 742-749

28 Lindstrom $J$ et al. Sustained reduction in the incidence of type 2 diabetes by lifestyle intervention: follow-up of the Finnish Diabetes Prevention Study. Lancet 2006; 368: 1673-1679

$29 \mathrm{Ou} \mathrm{HY}$ et al. Metformin increases insulin sensitivity and plasma betaendorphin in human subjects. Horm Metab Res 2006; 38: 106-111 
30 Ramachandran $A$ et al. The Indian Diabetes Prevention Programme shows that lifestyle modification and metformin prevent type 2 diabetes in Asian Indian subjects with impaired glucose tolerance (IDPP1). Diabetologia 2006; 49: 289-297

31 Torgerson JS et al. XENical in the prevention of diabetes in obese subjects (XENDOS) study: a randomized study of orlistat as an adjunct to lifestyle changes for the prevention of type 2 diabetes in obese patients. Diabetes Care 2004; 27: 155-161

32 Gerstein HC et al. Effect of rosiglitazone on the frequency of diabetes in patients with impaired glucose tolerance or impaired fasting glucose: a randomised controlled trial. Lancet 2006; 368: 1096-1105

33 Bosch J et al. Effect of ramipril on the incidence of diabetes. $\mathrm{N}$ Engl J Med 2006; 355: 1551-1562

34 Abuissa $\mathrm{H}$ et al. Angiotensin-converting enzyme inhibitors or angiotensin receptor blockers for prevention of type 2 diabetes: a metaanalysis of randomized clinical trials. J Am Coll Cardiol 2005; 46: 821-826

35 Chiasson JL, Brindisi MC, Rabasa-Lhoret R. The prevention of type 2 diabetes: what is the evidence? Minerva Endocrinol 2005; 30: 179-191

36 Watts $G$. Huge numbers must change lifestyle to prevent epidemic of diabetes. BMJ 2003; 327: 1365

37 Narayan KM, Bowman BA, Engelgau ME. Prevention of type 2 diabetes. BMJ 2001; 323: 63-64

38 Schwarz PE et al. Development of a diabetes prevention management program for clinical practice. Public Health Rep 2007; 122: 258-263

39 Schwarz PE et al. Prevention of type 2 diabetes: what challenges do we have to address? J Pub Health 2005; 13: 296-303

40 Schwarz PE. Targeted diabetes prevention in high risk groups: pro. Dtsch Med Wochenschr 2005; 130: 1103
41 Lindstrom J, Tuomilehto J. The Diabetes Risk Score: A practical tool to predict type 2 diabetes risk. Diabetes Care 2003; 26: 725-731

42 Saaristo T et al. Cross-sectional evaluation of the Finnish Diabetes Risk Score: a tool to identify undetected type 2 diabetes, abnormal glucose tolerance and metabolic syndrome. Diab Vasc Dis Res 2005; 2: 67-72

43 Schwarz PE et al. Prevention of type 2 diabetes in Germany. Ideas, evidence, implementation. Med Klin (Munich) 2006; 101: 730-736

44 Sartorelli DS et al. Primary prevention of type 2 diabetes through nutritional counseling. Diabetes Care 2004; 27: 3019

45 Schwarz PE et al. Diabetes prevention - from physiology to implementation. Horm Metab Res 2006; 38: 460-464

46 Lindstrom $J$ et al. High-fibre, low-fat diet predicts long-term weight loss and decreased type 2 diabetes risk: the Finnish Diabetes Prevention Study. Diabetologia 2006; 49: 912-920

47 Roubideaux $Y$ et al. Measuring the quality of diabetes care for older American Indians and Alaska natives. Am J Public Health 2004; 94: 60-65

48 Schwarz PE et al. Prevention of diabetes mellitus: The future of German diabetology. Hamostaseologie 2007; 27: 13-21

49 Hadaegh $F$ et al. Waist/height ratio as a better predictor of type 2 diabetes compared to body mass index in Tehranian adult men - a 3.6-year prospective study. Exp Clin Endocrinol Diabetes 2006; 114 (6): $310-315$

50 Wenying Y, Zhaojun Y. Obesity and hypertension as two anthropometric predictors for metabolic syndrome. Horm Metab Res 2006; 38: 469-470

51 Hanefeld $M$ et al. The Metabolic Syndrome - A Postprandial Disease? Horm Metab Res 2006; 38: 435-436 